\title{
Correction to: Causal effects of synthetic chemicals on mitochondrial deficits and diabetes pandemic
}

\author{
Wook-Ha Park ${ }^{1,2} \cdot$ Young-Chul Kang $^{1,2} \cdot$ Ying Piao $^{1,2} \cdot$ Daniel Hyungseok Pak $^{1,2,3}$. \\ Youngmi Kim Pak ${ }^{1,2}$
}

Published online: 29 August 2018

(C) The Pharmaceutical Society of Korea 2018

Correction to: Arch. Pharm. Res. (2013) 36:178-188

https://doi.org/10.1007/s12272-013-0022-9 should be corrected to $1.0 \mathrm{mM}$ instead of $0.5 \mathrm{mM}$. The corrected Fig. 4 should be:

We regret that there is an error in the labeling of Fig. 4 of the published original article. The concentration of $\mathrm{MPP}^{+}$

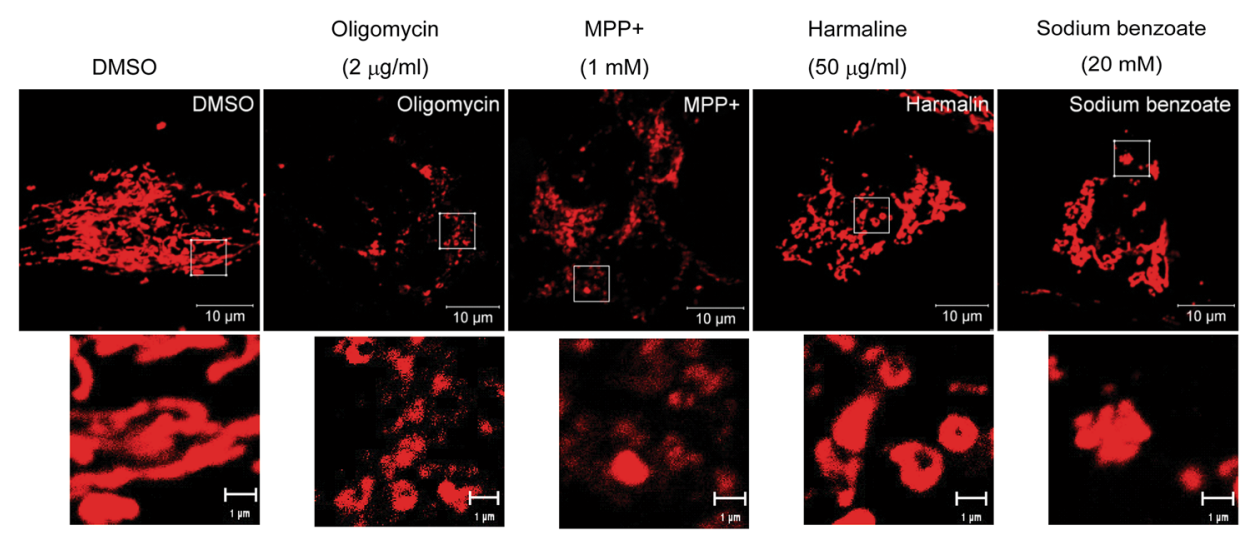

Fig. 4 Fixed confocal images of DsRed2-labeled mitochondria treated with various chemicals. All images are representations of SK-Hep1 cells transfected with DsRed2-mito vectors. Each image represents the effects of harmaline and sodium benzoate on mitochondrial morphology and dynamics. MPP ${ }^{+}$and oligomycin are positive controls, whereas DMSO is the negative control. Inset represents magnification of the boxed area. Scale bars are indicated on the images

The original article can be found online at https:// doi.org/10.1007/s12272-013-0022-9.

Youngmi Kim Pak

ykpak@khu.ac.kr

1 Department of Physiology, College of Medicine, Kyung Hee University, Seoul 130-701, Korea

2 Department of Neuroscience, College of Medicine, Kyung Hee University, Seoul 130-701, Korea

3 Department of Bioengineering, University of Illinois at Urbana-Champaign, Urbana, IL 61801, USA 